\title{
The MAIA candidate star HD 208727
}

\author{
T. Kallinger, P. Reegen, and W. W. Weiss \\ Institute for Astronomy (IfA), University of Vienna, Tuerkenschanzstrasse 17, 1180 Vienna, Austria \\ Received 13 March 2002 / Accepted 23 April 2002

\begin{abstract}
HD 208727 is a suspected member of the enigmatic MAIA variables and shows the largest yet observed amplitude in light variation for any of the potential members. Proven beyond doubt pulsation would pose a serious problem for stellar modelling and opacities, because either opacity source for driving pulsation has to be a different or new identified, yet unkown, pulsation mechanism. We conclude from our investigation that rotation is a more likely explanation for the light variability of HD 208727.
\end{abstract}

Key words. stars: variables: general - stars: fundamental parameters: radii - stars: individual: HD 208727

\section{Introduction}

Current knowledge of pulsation theory does not indicate pulsationally unstable stars in a small region of the Hertzsprung-Russell diagram between the blue edge of the $\delta$ Scuti instability strip and the red edge of the slowly pulsating B stars (SPB). There is no opacity "anomaly" known like the metal opacity bump for the SPB stars or the HeII bump for the $\delta$ Scuti stars which could drive pulsation in the given parameter space.

However, Struve (1955) suggested the existence of a group of variable stars in this region of the HR-diagram named after the prototype MAIA (HD 23408). According to Struve, this group has spectral and luminosity types between $\mathrm{B} 7 \mathrm{~V}$-III and $\mathrm{A} 2 \mathrm{~V}$-II and pulsation periods between 2 and $8 \mathrm{~h}$. Later Struve himself found no pulsation in MAIA and until now there is an ongoing dispute about the existence of the MAIA stars as a separate class of variables (Percy \& Wilson 2000, and references therein). Pulsation of a star in the MAIA area of the HR-diagram proven beyond doubt would pose a serious problem for theoretical astrophysics related to stellar models and opacities. Either opacity source for driving pulsation has to be a different or new identified, yet unknown, pulsation mechanism.

These arguments led us to investigate various members of the proposed group of MAIA variables (Weiss et al. 1998) and instrumental properties of the HIPPARCOS satellite (Kallinger \& Weiss 2002). Scholz et al. (1998) list 14 MAIA candidates and claim to have found indication for shortterm spectroscopic variations in two stars, $\gamma \mathrm{CRB}(\mathrm{A} 0 \mathrm{v})$ and $\gamma$ UMI (A3 III). With photometric data from the HIPPARCOS mission the list grew by 5 members (Aerts 2000, private communication), HD 4612, HD 46005,

Send offprint requests to: T. Kallinger,

e-mail: kallinger@astro.univie.ac.at
HD 106419, HD 121190, HD 175008 and HD 208727, which all seem to fulfill the period, spectral type and luminosity class criteria for MAIA stars.

During an $\mathrm{APT}^{1}{ }^{1}$ (Strassmeier et al. 1997) campaign we observed HD 208727 (v2173 CYG, HIP 108348).

\section{Observations and period search}

\subsection{HIPPARCOS}

The HIPPARCOs epoch photometry gives a light curve of HD 208727 with 133 data points at a photometric accuracy of $2.6 \mathrm{mmag}$ and it is listed in the HIPPARCOS catalog (ESA 1997) as a SPB star with a frequency of $3.16777 \mathrm{~d}^{-1}$. Using the DFT (Discrete Fourier Transform; Deeming 1975) based Fourier analysis program with a multi-sine fit option Period98 ${ }^{2}$ (Sperl 1998), we found a semi-amplitude of $21.8 \mathrm{mmag}$ and a photometric frequency of $3.16779 \mathrm{~d}^{-1}$ (period: $7.6 \mathrm{~h}$ ) which differs only in the 5 th digit of the frequency to the value given in the catalog. The corresponding phase diagram is shown in Fig. 1.

\section{2. $A P T$}

On Oct. 8, 2000, the APT took $6.3 \mathrm{~h}$ of Stromgren $b$ and $y$ measurements of HD 208727 yielding 70 data points. The comparison and check stars are listed in Table 1. The rms derived from comparison minus check star magnitudes is $1.34 \mathrm{mmag}$ in $b$ and $1.36 \mathrm{mmag}$ in $y$. In addition five previously taken APT monitoring data were available, distributed over 220 days.

\footnotetext{
1 Wolfgang-Amadeus: the University of Vienna twin automatic photoelectric telescope in Washington Camp, Arizona.

2 download from ftp://dsn.astro.univie.ac.at/pub/ Period98/
} 
We determined a harmonic period of $7.6 \mathrm{~h}$ with an amplitude of $30 \mathrm{mmag}$ in our data. After prewhitening with this period the residuals do not show any significant periodicity remaining. For $(b-y)$ we obtained $-0.036 \pm 0.003$ (SIMBAD : -0.03). The phase diagram of the folded light curve (Fig. 2) shows good phase agreement between the monitoring data points and the $6.3 \mathrm{~h}$ light curve, thus confirming the period determined from the HIPPARCOS data. The $10 \mathrm{mmag}$ offset between light curve and monitoring data is most probably due to the use of default extinction coefficients for the latter.

\section{Discussion}

From the HIPPARCOS parallax of $3.31 \pm 0.55$ mas and $V=6.42$ (Deutschmann et al. 1976) we obtained $M_{V}=-0.98$. Cowley et al. (1969) classify HD 208727 as $\mathrm{B} 8 \mathrm{~V}$ in their catalogue of spectral classification of bright stars. Our value of $(b-y)$ in combination with $\beta=2.758, m_{1}=0.121, c_{1}=0.609$ from SIMBAD yields $T_{\text {eff }}=13068 \mathrm{~K}$, based on the calibration of the Stromgren system by Moon \& Dworetsky (1985) and the correction by Napiwotzki et al. (1993). This temperature corresponds to a B8.3 V star (Zombeck 1990), which is in good agreement with Cowley et al. (1969). The bolometric correction for a MS star of this temperature is -0.981 (Reed 1998) which results in an estimated radius for HD 208727 of $1.73 R_{\odot}$ and a rotation period $P_{\text {rot }}$ according to

$$
\begin{aligned}
& R / R_{\odot}=2.964 \times 10^{8} \cdot T_{\mathrm{eff}}^{-2} \times 10^{-0.2\left(M_{V}-B C\right)} \\
& P_{\text {rot }} \cdot \sin i^{-1}(h)=1212 \cdot\left(R / R_{\odot}\right) \cdot(v \sin i)^{-1}
\end{aligned}
$$

With a $v \sin i$ of $240 \mathrm{~km} \mathrm{~s}^{-1}$, taken from Abt \& Morrell (1995), we can estimate $P_{\text {rot }} \sin i^{-1}$ ranging from 7.4 to $10.7 \mathrm{~h}$, taking the errors of the various input parameters into account. The observed period of $7.6 \mathrm{~h}$ would be fully consistent with a rotational modulation of the light curve of HD 208727 with an angle of inclination of $60^{\circ}$ (for the most probable value of $P_{\text {rot }} \sin i^{-1}=8.76 \mathrm{~h}$ ).

What about the speculation that HD 208727 is a SPB star (ESA 1997), reiterated by Adelman et al. (2000)? First, the period of $7.6 \mathrm{~h}$ is extremely short in respect of the periods found for well established SPB stars (Percy \& Wilson 2000) which range from $12 \mathrm{~h}-2$ days, in agreement with values given by Aerts et al. (2000) and Pamyatnykh (1999). The latter gives in his Fig. $510 \mathrm{~h}$ as shortest yet observed SPB pulsation period. A stability analysis of Pamyatnykh (2002, private communication) for a stellar model $\left(M / M_{\odot}=3.25, \log T_{\text {eff }}=4.1, \log g=4.21\right.$, $\left.v_{\text {rot }}=239 \mathrm{~km} \mathrm{~s}^{-1}\right)$, hence with very similar parameters as for HD 208727, indicate no unstable p-modes in the given period range and the shortest unstable $g$-mode $(\ell=2$, $n=12$ ) with a period of $11.8 \mathrm{~h}$.

Since the angle of expectation for stellar rotation axes uniformly distributed in space is $57^{\circ}$, we conclude that rotation rather than pulsation causes the photometric variability observed for this MAIA candidate star and no
Table 1. APT campaign stars.

\begin{tabular}{llccc}
\hline \hline Typ & HD & $\begin{array}{c}V \\
{[\mathrm{mag}]}\end{array}$ & $\begin{array}{c}\text { spectral } \\
\text { type }\end{array}$ & $\begin{array}{c}B-V \\
{[\mathrm{mag}]}\end{array}$ \\
\hline Variable & HD 208727 & 6.4 & $B 8 V$ & -0.1 \\
Comparison & HD 208394 & 7.4 & $A 2$ & 0.2 \\
Check & HD 208441 & 7.8 & $A 0$ & 0.04 \\
\hline
\end{tabular}

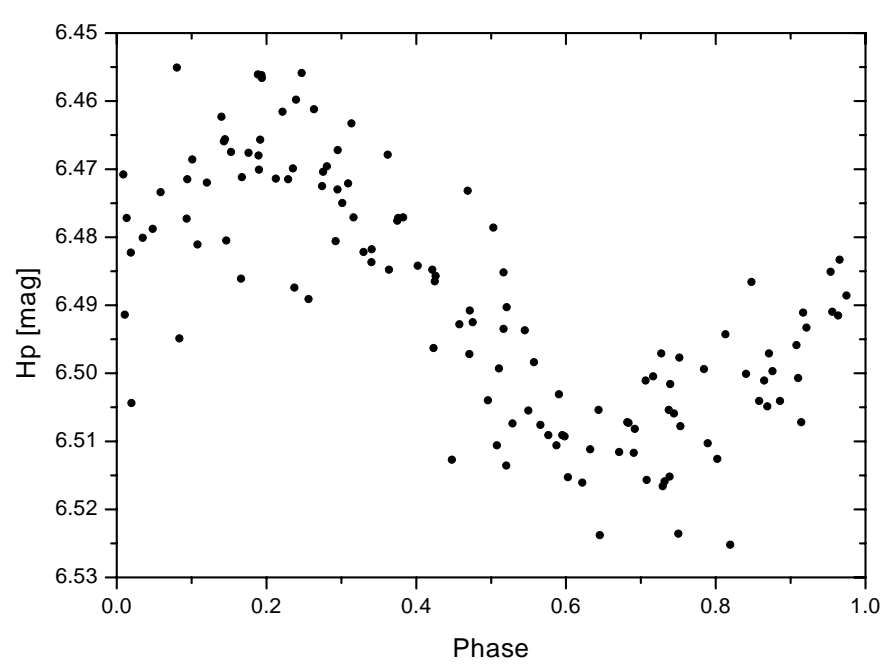

Fig. 1. Phase diagram based on HIPPARCOS epoch photometry of HD 208727 for a $7.6 \mathrm{~h}$ period. The obtained amplitude is $21.8 \mathrm{mmag}$.

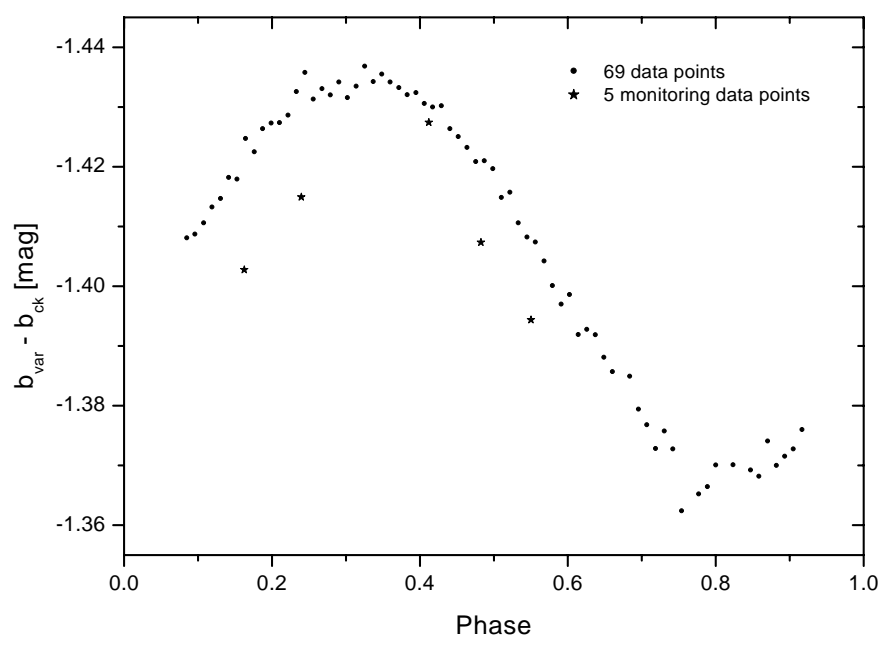

Fig. 2. Phase diagram based on APT photometry (Stromgren $b$ ) of HD 208727 for a $7.6 \mathrm{~h}$ period. The obtained amplitude is $30 \mathrm{mmag}$.

yet unknown physical processes are required to explain the variability of HD 208727. Spectroscopic observations should indicate atmospheric inhomogeneities which would be required for a rotational modulation in brightness. However, the large observed $v \sin i$ will make this proof very difficult.

Acknowledgements. Financial support was received from the Bundesministerium für Bildung, Wissenschaft und Kultur (project EXTRACTOR) and the Austrian Fonds zur Förderung der wissenschaftlichen Forschung (projects P14984 
and P14546). We also acknowledge comments from an unknown referee which helped to improve the manuscript.

\section{References}

Abt, H. A., \& Morrell, N. I. 1995, ApJS, 99, 135

Adelman, S. J., Gentry, M. L., \& Suidiana, I. M. 2000, IBVS, 4968

Aerts, C., De Cat, P., De Ridder, J., et al. 2000, in The Impact of Large-Scale Surveys on Pulsating Star Research, IAU Coll. 176, ed. L. Szabados, \& D. Kurtz, ASP Conf. Ser., 203, 395

Cowley, A., Cowley, C., Jaschek, M., \& Jaschek, C. 1969, AJ, 74,375

Deeming, T. J. 1975, Ap\&SS, 36, 137

Deutschman, W. A., Davis, R. J., \& Schild, R. E. 1976, ApJS, 30, 97

ESA 1997, The Hipparcos and Tycho Catalogue, ESA SP-1200
Kallinger, T., \& Weiss, W. W. 2002, A\&A, 385, 533

Moon, T. T., \& Dworetsky, M. M. 1985, MNRAS, 217, 305

Napiwotzki, R., Schönberger, D., \& Wenske, V. 1993, A\&A, 268,635

Pamyatnykh, A. A. 1999, Acta Astron., 49, 119

Percy, J. R., \& Wilson, J. B. 2000, PASP, 112, 846

Rogers, N. Y. 1995, Comm. Astroseismology (Vienna), 78, 1

Reed, C. 1998, JRASC, 669, 36

Scholz, G., Lehmann, H., Hildebrandt, G., Panov, K., \& Illiev, L. 1998, A\&A, 337, 447

Sperl, M. 1998, Comm. Astroseismology (Vienna), 111, 1

Strassmeier, K. G., Boyd, L. J., Epand, D. H., \& Granzer, T. 1997, PASP, 109, 697

Struve, O. 1955, Sky and Telescope, 14, 461

Weiss, W. W., Kuschnig, R., Mkrtichian, D. E., et al. 1998, A\&A, 338, 919

Zombeck, M. V. 1990, Handbook of Space Astronomy and Astrophysics (Cambridge University Press) 